www.jmscr.igmpublication.org Impact Factor 5.244

Index Copernicus Value: 5.88 ISSN (e)-2347-176x ISSN (p) 2455-0450 crossref DOI: http://dx.doi.org/10.18535/jmscr/v4i6.68

Journal Of Medical Science And Clinical Research

\title{
Role of Hormones in Orthodontics: A Review
}

\author{
(Review Article)
}

\section{Authors \\ Dr Sourabh Jindal ${ }^{1}$ Dr Amit Khera ${ }^{2}$, Pradeep Raghav ${ }^{3}$, Dr Ayush Jain ${ }^{4}$, Dr Gourav Popli ${ }^{5}$, Dr Navana Gupta ${ }^{6}$}

${ }^{1}$ Postgraduate Student, Dept of Orthodontics and Dentofacial Orthopaedics, Subharti Dental College, Meerut

${ }^{2}$ Reader, Department of Orthodontics and Dentofacial Orthopaedics, Subharti Dental College, Meerut

${ }^{3}$ Professor and Head, Dept of Orthodontics and Dentofacial Orthopaedics, Subharti Dental College, Meerut

${ }^{4}$ Postgraduate Student, Dept of Orthodontics and Dentofacial Orthopaedics, Subharti Dental College, Meerut

${ }_{5}^{5}$ Senior Lecturer, Department of Oral and Maxillofacial Surgery, Subharti Dental College, Meerut

${ }^{6}$ Postgraduate Student, Dept of Orthodontics and Dentofacial Orthopaedics, Subharti Dental College, Meerut

Corresponding Author

\section{Dr Gourav Popli}

Senior Lecturer, Department of Oral and Maxillofacial Surgery

Subharti Dental college, Swami Vivekanand Subharti University, Meerut

Email:-drgp0250@gmail.com, Phone No:- +918475991147

\section{ABSTRACT}

Hormones are tiny chemical messengers located inside of our body. Different hormones perform specific roles inside of our body. Some of these hormones work quickly to start or stop a process, and some will continually work over the course of a long period of time to perform their necessary jobs. On the other hand, the biology of orthodontic tooth movement comprises the study of cellular, biochemical and molecular phenomena occurring in the periodontal ligament and alveolar bone. The bone remodeling that occurs in the orthodontic movement is a dynamic process requiring coordinated cellular activities between osteoblasts, osteocytes and osteoclasts, in order to maintain the mineral tissue homeostasis. All these activities are regulated by chemical mediators, of cellular and plasmatic origin mainly hormones. Authors have emphasised on the role of various endocrine and paracrine hormones on the orthodontic tooth movements through the literature review.

\section{INTRODUCTION}

The activities of various organs in our body are controlled by two systems namely, nervous system and endocrine system. ${ }^{1}$ Most of the functions of nervous system are executed by hormonal substances, and endocrine functions are controlled by nervous system.
The endocrine system constitutes endocrine glands which are situated in different parts of body. ${ }^{2}$ The functions of these glands are mediated by chemical substances which are called chemical messengers or chemical mediators or first messengers or hormones. The endocrine glands are also called as ductless glands because the 
hormones secreted by them are directly release into blood. ${ }^{3}$

In June 1905, Ernest Starling, a professor of physiology at University College London, UK, first used the word 'hormone' ${ }^{4}$. Starling (1905) defined the word, derived from the Greek meaning 'to arouse or excite', as 'the chemical messengers which speeding from cell to cell along the blood stream, may coordinate the activities and growth of different parts of the body".

Orthodontic tooth movement results from the response of the periodontal tissue to the orthodontic force, which leads to modeling and remodeling of the surrounding alveolar bone. The response is considered to occur through the activation of specific signaling pathways, many of which are known, all acting to ultimately result in tooth movement. The rate at which tooth movement occurs depends on the ability of these pathways to realize bone metabolism by the two main cell types responsible for tooth movement: osteoblasts and osteoclasts. 3,5,6

Hormones have an important influence on the rate of tooth movement, and information on their consumption is essential to adequately discuss treatment planning with patients. This is especially important in dentistry because many of the patients attending dental clinics face stressful situations. $^{7}$

Awareness is therefore necessary on the risks and difficulties that may arise during the dental and orthodontic management of patients with endocrine disorders and most common oral manifestations. $^{2}$

\section{DISCUSSION}

\section{Growth Hormone (Gh)}

$\mathrm{GH}$ is a protein hormone, secreted by the acidophils of the anterior pituitary gland. GH secretion is pulsatile, secretory bursts occurring especially at early hours of sleep and throughout the night. GH has no specific target organ. It is an anabolic hormone to which every organ system responds. Apparently, it has no direct action upon bones, acting through a substance called somatomedin. GH stimulates the liver to secrete somatomedin and is the main regulator of childhood and adolescent growth.

Growth hormone, also called somatotropic hormone or somatotropin, is a small protein molecule that contains 191 amino acids in a single chain and has a molecular weight of 22,005. It causes growth of almost all tissues of the body that are capable of growing. It promotes increased sizes of the cells and increased mitosis, with development of greater numbers of cells and specific differentiation of certain types of cells such as bone growth cells and early muscle cells. ${ }^{1}$

\section{Metabolic Effects ${ }^{7}$}

Aside from its general effect in causing growth, growth hormone has multiple specific metabolic effects, including

(1) Increased rate of protein synthesis in most cells of the body;

(2) Increased mobilization of fatty acids from adipose tissue, increased free fatty acids in the blood, and increased use of fatty acids for energy; and

(3) Decreased rate of glucose utilization throughout the body.

\section{Growth Hormone Effect on Cartilage and Bone Growth}

(1) Increased deposition of protein by the chondrocytic and osteogenic cells that cause bone growth.

(2) Increased rate of reproduction of these cells.

(3) A specific effect of converting chondrocytes into osteogenic cells, thus causing deposition of new bone. ${ }^{8}$

\section{Effects of Growth Hormone on Craniofacial Growth:}

With Idiopathic Growth Hormone Deficiency, the length and depth of the face are inappropriately small for the child's age, with the face maintaining childlike convexity. Many studies have reported mandibular total length $(\mathrm{Gn}-\mathrm{Cd})$ is 
reduced, primarily as a result of the small ramus height (Cd- Go).

In addition, the maxilla is significantly reduced, and there may be a comparable degree of reduction in the mandible. The maxilla is often retrognathic but is affected less than the mandible. Concerning cranial base size, many studies have reported that the posterior cranial base length is smaller than the anterior cranial base (N-S) length. By contrast, facial convexity decreases with GH replacement therapy, and its main effect seems to be on condylar growth ${ }^{9}$.

Another study reported that growth in the $\mathrm{Gn}-\mathrm{Cd}$ and lower facial height (ANS-Me) are accelerated, whereas the cranial base length changes minimally. Cantu et al found that catch-up growth with GH therapy affects the anterior facial height, posterior facial height, and posterior cranial base ${ }^{2}$.

\section{Effect on Dental Development}

Dental delay is always less pronounced than height or bone delay. Dentition seems to be harmoniously delayed, so that all studied components of dental development (primary root resorption, secondary tooth formation and eruptive movement) display the same degree of retardation.GH influence on growth starts after 9 months of age, so that the effect on the growth of primary teeth is very little known. ${ }^{7}$

\section{Prostaglandins (Pgs)}

PROSTAGLANDINS are a group of chemical messengers belonging to a family of hormones called eicosanoids. These are paracrine hormones, i.e. they act only on cells near the point of hormone synthesis instead of being transported via blood to act on cells in other tissues or organs, and they have a variety of dramatic effects on vertebrate tissues. All eicosanoids are derived from arachidonic acid, from which they take their general name. The three major classes of eicosanoids are prostaglandins, thromboxanes and leukotrienes. Thromboxanes are produced in the human body by platelets and act in blood clot formation, while leukotrienes are involved in inflammation and their overproduction causes asthmatic attacks. ${ }^{18}$ Prostaglandins act in many tissues by regulating the synthesis of cyclic AMP. As cyclic AMP mediates the actions of diverse hormones, prostaglandins affect a wide range of cellular and tissue functions:

(1) They stimulate contraction of the smooth muscle of the uterus.

(2) They affect blood flow, sleep cycle and response to hormones such as adrenaline and glucagon.

(3) They elevate body temperature, cause inflammation and pain.

\section{Prostaglandins and orthodontics}

Yamasaki and associates conducted experiments on rats to investigate whether the synthesis of prostaglandins is induced by orthodontic force, and whether exogenous prostaglandins can produce bone resorption similar to orthodontic force. They reported that the application of orthodontic force did indeed cause increased synthesis of prostaglandins, which in turn stimulates osteoclastic bone resorption.

Von Euler, who first discovered the compound in human semen and believed the prostate gland to be the main source of this chemical substance, introduced the term prostaglandin. However, it was later discovered that most cell types in the body produce prostaglandins ${ }^{11}$.

Harell et al suggested that prostaglandins are important mediators of mechanical stress. This finding was followed by the work of Yamasaki et al, who found an increase in osteoclast numbers after a local injection of prostaglandins into the paradental tissues of rodents. This association was demonstrated by the reduced rate of tooth movement after the administration of indomethocin, an anti-inflammatory agent and a specific inhibitor of prostaglandin synthesis. ${ }^{12}$

Clinical and animal studies by various authors have identified the role of prostaglandins (PGE1 and PGE2) in stimulating bone resorption. They have reported a direct action of prostaglandins on osteoclasts in increasing their numbers and their 
capacity to form a ruffled border and effect bone resorption. Like other bone resorbing agents, PGE2 also stimulates osteoblastic cell differentiation and new bone formation, coupling bone resorption in vitro. Studies have also identified other agents such as growth factors (platelet-derived growth factors), hormones (parathormone $[\mathrm{PTH}]$ ), and interleukins or other cytokines that induce PGE2 production, to effect bone remodeling and tooth movement.

Chumbley et al reported that Indomethacin, an inhibitor of prostaglandin synthesis, also inhibited orthodontic tooth movement. Yamasaki et al studied the effects of PGE1 administration on orthodontic tooth movement in humans. This study was performed in two phases. In the first phase, buccal movement of the first premolars scheduled for extraction was examined with and without PGE1 administration. In the second stage, PGE1 was administered in canine retraction for up to three weeks in first premolar extraction cases. In both phases, the rate of tooth movement was doubled compared to control sides. Lee et al also reported that systemic intravenous administration of PGE1 was more effective and produced more bone resorption than local injection ${ }^{13}$. Bhalajhi and Shetty studied the effect of exogenous administration of PGE2 in young rabbits and reported an increase in the rate of tooth movement clinically and an increase in the number of osteoclasts and resorption lacunae, microscopically. The two major Drawbacks associated with the use of prostaglandins as reported by them are: pain reaction and the need for frequent administration because of rapid metabolism of PGE2 in the lung ${ }^{14}$.

\section{Corticosteroids}

Corticosteroids are immunosuppressive and antiinflammatory agents, widely used to treat pathological processes in medical and dental practice, in such a way that patients under orthodontic treatment may present variations in normal bone remodeling due to the use of these drugs. 15
Hyperglucocorticoidism leads to a short stature and developed bone maturation but increases relative weight. Very small amounts given a medication can decrease growth rate. Skeletal IGFI synthesis is decreased by cortisol, which has an inhibitory effect on bone collagen synthesis. In the process of tooth eruption, however, cortisone has a special effect. Eruption rate is accelerated.

\section{Effects on bone and tooth movement}

Evidence indicates that the main effect of corticosteroids on bone tissue is direct inhibition of osteoblastic function and thus the decrease of total bone formation. Decrease in bone formation is due to elevated parathyroid hormone levels caused by inhibition of intestinal calcium absorption which are induced by corticosteroids. Corticosteroids increase the rate of tooth movement, and since new bone formation can be difficult in treated patients, they decrease the stability of tooth movement and stability of orthodontic treatment in general. ${ }^{12}$ When they are used for longer periods of time, the main side effect is osteoporosis. It has been demonstrated in animal models with this type of osteoporosis that the rate of active tooth movement is greater, but tooth movement is less stable since little bone is present and there is no indication of bone formation.

\section{Estrogens}

Estrogen is considered to be the most important hormone to affect bone metabolism in women. It controls bone remodeling during reproductive life, and maintenance of maximum bone mass after menarche. Estrogen decreases the rate of bone resorption. Estrogen inhibits the production of various cytokines, mainly interleukin-1 (IL-1), (TNF-a) tumor necrosis factor-alpha, and interleukin-6(IL-6), which are involved in bone resorption by stimulating osteoclast formation and osteoclastic bone resorption. Deficiency in estrogen seems to be responsible for the secondary hyperparathyroidism found in postmenopausal women, as they also inhibit osteoblasts 
responsiveness to parathyroid hormone. Estrogens do not have any anabolic effects on bone tissue; they directly stimulate the bone forming activity of osteoblasts ${ }^{16}$.

\section{Effect on tooth movement}

Studies have shown that Estrogen decreases the velocity of tooth movement. Oral contraceptives taken for long periods of time can influence the rate of tooth movement. Androgens also inhibit bone resorption and modulate the growth of the muscular system and may affect the length and results of orthodontic treatment. Tooth movement occurs as a consequence of periodontal tissue remodeling when force is applied to teeth. The process of periodontal tissue remodeling involves the following: stretching of the periodontal ligament and deposition of the new alveolar bone at the tension region; and compression of the periodontal ligament and the resorption of the alveolar bone at the pressure region. The rate of periodontal tissue remodeling is influenced by various factors such as the estrogen level. Previous studies have shown the presence of estrogen receptors in the periodontal tissue, indicating that this tissue is targeted by estrogen. Estrogen influences the composition and degradation of collagen fibers in the periodontal ligaments and the remodeling of the alveolar bones. While estrogen influences the deposition and cross-linking of collagen fibers, it also enhances the alkaline phosphatase (ALP) activity and the secretion of osteocalcin (OCN) and osteoprotegerin (OPG) in the periodontal ligament cells (PDLCs) ${ }^{17}$. Rate of tooth movement is tied up with activity of osteoclasts. Estrogen can inhibit activity of osteoclasts in a direct or indirect manner, thereby modulating bone resorption. Low estrogen levels stimulate the osteoblastic production of bone resorption related factors, such as interleukin-1 and -6, tumor necrosis factoralpha (TNF-a), macrophage colony-stimulating factor (M-CSF); these factors may induce bone loss by affecting the differentiation and activity of osteoclasts. Estrogen inhibits tooth movement by increasing the bone mineral content and bone mass and by reducing the bone resorption rate. Several studies have shown that estrogen deficiency accelerated tooth movement ${ }^{5}$.

Estrogen directly stimulates the bone-forming activity of osteoblasts, so it is reasonable to expect a decrease of the velocity of orthodontic tooth movement. Androgens also inhibit bone resorption and modulate the growth of the muscular system. Thus, the excessive use of these drugs by athletes, in an attempt to achieve better athletic scores, may affect the length and the results of orthodontic treatment ${ }^{18}$.

\section{Thyroxine Hormones (Th)}

Thyroid hormones are recommended for the treatment of hypothyroidism and used after thyroidectomy in substitutive therapy. Thyroxin administration lead to increased bone remodeling, increased bone resorptive activity, and reduced bone density. Effects on bone tissue may be related to the augmentation of interleukin-1 (IL1B) production that thyroid hormones induce at low concentrations, cytokine stimulate osteoclast formation and osteoclastic bone resorption. The speed of orthodontic tooth movement increases in patients undergoing such medication. Low-dosage and short-term thyroxin administrations are reported to lower the frequency of "force induced" root resorption. Decrease in resorption may be correlated to a change in bone remodeling process and a reinforcement of the protection of the cementum and dentin to "force induced" osteoclastic resorption $^{21}$.

Thyroid disorders commonly affect craniofacial and dental structures. The dental and craniofacial retardation manifested under prolonged hypothyroid conditions differs from the isolated lack of GH. The main difference is the cranial vault, which shows growth retardation in hypothyroidism, and reduced facial height in children with prolonged untreated hypothyroidism. Thyroxin administration seems to lead to increased bone remodeling, increased bone resorptive activity and reduced bone density. 
Thyroid hormones increase osteoclastic bone resorption in neonatal mouse calvarium by stimulation of prostaglandin synthesis ${ }^{21}$.

\section{Parathyroid hormone (PTH)}

Parathyroid hormone provides a powerful mechanism for controlling extracellular calcium and phosphate concentrations by regulating intestinal reabsorption, renal excretion, and exchange between the extracellular fluid and bone of these ions. Excess activity of the parathyroid gland causes rapid absorption of calcium salts from the bones, with resultant hypercalcemia in the extracellular fluid; conversely, hypofunction of the parathyroid glands causes hypocalcemia, often with resultant tetany ${ }^{22}$.

Parathyroid hormone is produced by parathyroid glands to regulate serum calcium concentration. In kidneys, PTH increases renal calcium resorption and stimulates the excretion of urinary phosphate. In bone, PTH can induce a rapid release of calcium, but also mediates longer term changes by acting directly on osteoblasts \& indirectly on osteoclasts. PTH affects osteoblasts' cellular metabolic activity, gene transcriptional activity, and multiple protease secretion. Its effects on osteoclasts occur through the production of RANK-L, a protein that plays a crucial role in osteoclast formation and activity.

\section{Effects of PTH on tooth movement}

Parathyroid hormone, as a major regulator of calcium and phosphate homeostasis, has gained particular attention for its paradoxical effects on bone metabolism. Relative studies have confirmed that parathyroid hormone could stimulate both osteoclast-mediated bone resorption and osteoblast-mediated bone formation, therefore accelerating the bone turnover rate. The ultimate effect of parathyroid hormone on bone remodeling is determined by the administration protocol. Continuous infusion of parathyroid hormone results in a catabolic effect, whereas intermittent injection leads to an anabolic effect. Intermittent low-dose parathyroid hormone analogs have been widely administered in the clinical treatment of osteoporosis. Previous studies have investigated the effect of different administration patterns of parathyroid hormone and the results suggested that systemic continuous infusion or local chronic application of parathyroid hormone could accelerate tooth movement through enhancement of alveolar bone resorption, whereas long-term intermittent injection of parathyroid hormone facilitated periodontal repair of bone or root resorption after orthodontic tooth movement through activation of osteoblastic cells ${ }^{23}$.

Salazar et al evaluated the tooth movement with intermittent parathyroid hormone injections mentioned that unlike other osteoporosis treatment drugs such as bisphosphonates, calcitonin, and calcium with vitamin $\mathrm{D}$, which diminish bone resorption, parathyroid hormones stimulated osteoblast function with no interference in osteoclast activity and facilitated bone remodeling. This could explain the acceleration effect of intermittent parathyroid hormone. The precise mechanism underlying the effect of intermittent parathyroid hormone on bone metabolism has not been clarified yet. However, it is verified that under intermittent parathyroid hormone administration, both osteoblast and osteoclast activities are stimulated. The anabolic effect of intermittent parathyroid hormone in the clinical treatment of osteoporosis involves not only osteoblastic bone formation, but also osteoclastic bone resorption. The ultimate increase of bone density is achieved through the "anabolic window," which means that bone formation is greater than bone resorption during the first 6 to 18 months. Actually, some researchers suppose that active osteoclastic resorption is necessary for the effect of the parathyroid hormone on bone formation in a remodeling system. Intermittent parathyroid hormone administration, results in an increase in osteoclastic resorptive activity. In turn, the resorptive activity increases the release of osteogenic growth factors from bone matrix and osteoclasts, and it stimulates bone remodeling ${ }^{7,8}$. 


\section{Calcitonin}

Calcitonin, a peptide hormone secreted by the thyroid gland, tends to decrease plasma calcium concentration and, in general, has effects opposite to those of PTH. However, the quantitative role of calcitonin is far less than that of PTH in regulating calcium ion concentration. Synthesis and secretion of calcitonin occur in the parafollicular cells, or C cells, lying in the interstitial fluid between the follicles of the thyroid gland. These cells constitute only about 0.1 per cent of the human thyroid gland ${ }^{24}$.

\section{Increased Plasma Calcium Concentration} Stimulates Calcitonin Secretion. The primary stimulus for calcitonin secretion is increased plasma calcium ion concentration. This contrasts with PTH secretion, which is stimulated by decreased calcium concentration. An increase in plasma calcium concentration of about 10 per cent causes an immediate two fold or more increase in the rate of secretion of calcitonin. This provides a second hormonal feedback mechanism for controlling the plasma calcium ion concentration, but one that is relatively weak and works in a way opposite that of the PTH system. Calcitonin inhibits proximal tubular calcium and phosphate reabsorption by direct action on kidney. Calcitonin is used in the treatment of hypercalcemia, osteoporosis and Paget's disease of bone ${ }^{25}$.

\section{Effects of Calcitonin on bone and tooth movement}

Calcitonin inhibits bone resorption by direct action on osteoclasts decreasing their ruffled surface which forms contact with resorptive pit. It also stimulates the activity of osteoblasts. Because of its physiological role, it is considered to inhibit the tooth movement, consequently delay in orthodontic treatment can be expected.

\section{Vitamin D}

Vitamin D has a potent effect to increase calcium absorption from the intestinal tract; it also has important effects on both bone deposition and bone absorption. However, vitamin D itself is not the active substance that actually causes these effects. Instead, vitamin D must first be converted through a succession of reactions in the liver and the kidneys to the final active product, 1, 25dihydroxycholecalciferol shows the succession of steps that lead to the formation of this substance from vitamin $\mathrm{D}^{26}$.

\section{Actions of Vitamin D}

Promotes intestinal calcium absorption.

Promotes phosphate absorption from intestines.

Decreases renal calcium and phosphate excretion.

Vitamin-D and its most active metabolite, vitamin-D3, together with parathyroid hormone and Calcitonin, regulate the amount of calcium and phosphorus in the human organism. It promotes intestinal $\mathrm{Ca}+2$ and PO4-3 absorption. Vitamin- D3 increases bone mass and thus reduce fractures in osteoporosis patients. Considering its beneficial effects on bone tissue, it may be assumed that it inhibits tooth movement.

\section{REFERENCES}

1. Brasel JA, Blizzard RM. Textbook of Endocrinology. 5th ed. Philadelphia, Pa: WB Sauders; 1974.

2. Thilander B. Basic mechanisms in craniofacial growth. Acta Odontol Scand. 1995; 53:144-151.

3. Pirinen S. Endocrine regulation of craniofacial growth. Acta Odontol Scand. 1995; 53:179-18

4. C.C chatterji text book of physiology volume 2 page 4(2-9)

5. Funatsu M, Sato K, Mitani H. Effects of growth hormone on craniofacial growth. Angle Orthod. 2006; 76:970-977.

6. Bevis RR, Hayles AB, Isaacson RJ, Sather AH. Facial growth response to human growth hormone in hypopituitary dwarfs. Angle Orthod. 1977; 47:193-205.

7. Sunil Kumar Khare, Rajendra Gupta, Amit Prakash. Hormones and their Clinical 
Consideration in Orthodontics. Indian J Dent Adv 2013; 5(1): 1120-1124

8. George Litsas*.Growth Hormone and Craniofacial Tissues. An update. The Open Dentistry Journal, 2015, 9, 1-8

9. Gupta Akshay1, Sharma Rakesh2, Kumar Piush3 \& Chandra Pavan Kumar4. Effect of pharmacological agents on orthodontic tooth movement. J Pharm Biomed Sci. 2013, March; 28 (28): 688-694

10. Ashima Valiathan* and Siddhartha Dhar. Prostaglandins and enhanced orthodontic tooth movement: In search of the silver bullet. CURRENT SCIENCE, VOL. 90, NO. 3, 10 FEBRUARY 2006

11. Saladin. Anatomy and Physiology, The unity of form and function.

12. Amit Prakash, Prabhuraj Sabarad, Sonali Rai Hormones and their Clinical Consideration in Orthodontics. Indian $\mathbf{J}$ Dent Adv 2013; 5(1): 1120-1124

13. LESLIE P. GARTNER, JAMES L. HIATT, Color Atlas and Text ofHistology, sixth edition.

14. Victor P. Eroschenko, diFiore's Atlas of Histology with functional correlations, eleventh edition

15. Knop LAH, Shintcovsk RL, Retamoso LB, Grégio AMT, Tanaka O. The action of corticosteroids on orthodontic tooth movement: A literature review. Dental Press J Orthod. 2012 NovDec;17(6):20.e1-5.

16. Minayo Funatsua; Koshi Satob; Hideo Mitanic Effects of Growth Hormone on Craniofacial Growth. Angle Orthodontist, Vol 76, No 6,2006. DOI:10.2319/011905-1

17. Seunghye Kim, Seong Oh Kim, Chul Hee Kim*, Jae-Ho Lee, Heung-Kyu Son. The Effect of Hyperthyroidism on The Rate Of Orthodontic Tooth MOVEMENT. J Korean Acad Pediatr Dent 37(2) 2010.
18. Tarvade SM, Daokar SG. Osteoporosis and orthodontics: A Review. Sci J Dent 2014;1:26-29.

19. KKnop LAH, Shintcovsk RL, Retamoso LB, Grégio AMT, Tanaka O. The action of corticosteroids on orthodontic tooth movement: A literature review. Dental Press J Orthod. 2012 NovDec;17(6):20.e1-5.

20. Richard S. Masellaa and Malcolm Meisterb Fort Lauderdale, Fla. Current concepts in the biology of orthodontic tooth movement. Am J Orthod Dentofacial Orthop 2006;129:458-68)

21. Hector F DeLuca. Overview of general physiologic features and functions of vitamin D1-4. Am J Clin Nutr 2004;80(suppl):1689S-96S. Printed in USA. (C) 2004 American Society for Clinical Nutrition.

22. Colin K. L. Ong,; Laurence J. Walsh, Douglas Harbrow, Aart A. R. Taverne, Anne L. Symonse Orthodontic Tooth Movement in the Prednisolone-Treated Rat. Angle Orthodontist, V.70, No 2, 2000

23. VP. Drug Induced Orthodontic Tooth Movement- A Review.J Adv Med Dent Scie Res 2015;3(1):191-195.

24. Niels Møller and Jens Otto Lunde Jørgensen. Effects of Growth Hormone on Glucose, Lipid,and Protein Metabolism in Human Subjects. Endocrine Reviews, April 2009, 30(2):152-177.

25. Vigneshwar Sambandam1, Prasanna Neelakantan2*. Steroids in Dentistry - A Review. Int. J. Pharm. Sci. Rev. Res., 22(2), Sep - Oct 2013; no 44, 240-245.

26. Kamatchi Diravidamani, Sathesh Kumar, Sivalingam,Viviek Agarwal. Drugs Influencing Orthodontic Tooth Movement: An Overall Review. J Pharm Bioallsci 2012;4:299-303. 